\title{
Progressive high-load strength training compared with general low-load exercises in patients with rotator cuff tendinopathy: study protocol for a randomised controlled trial
}

Kim G Ingwersen ${ }^{1,2^{*}}$, Robin Christensen ${ }^{3}$, Lilli Sørensen ${ }^{4}$, Hans RI Jørgensen ${ }^{5}$, Steen Lund Jensen ${ }^{6}$, Sten Rasmussen ${ }^{7,8}$, Karen Søgaard ${ }^{1}$ and Birgit Juul-Kristensen ${ }^{1,9}$

\begin{abstract}
Background: Shoulder pain is the third most common musculoskeletal disorder, often affecting people's daily living and work capacity. The most common shoulder disorder is the subacromial impingement syndrome (SIS) which, among other pathophysiological changes, is often characterised by rotator cuff tendinopathy. Exercise is often considered the primary treatment option for rotator cuff tendinopathy, but there is no consensus on which exercise strategy is the most effective. As eccentric and high-load strength training have been shown to have a positive effect on patella and Achilles tendinopathy, the aim of this trial is to compare the efficacy of progressive high-load exercises with traditional low-load exercises in patients with rotator cuff tendinopathy.

Methods/Design: The current study is a randomised, participant- and assessor-blinded, controlled multicentre trial. A total of 260 patients with rotator cuff tendinopathy will be recruited from three outpatient shoulder departments in Denmark, and randomised to either 12 weeks of progressive high-load strength training or to general low-load exercises. Patients will receive six individually guided exercise sessions with a physiotherapist and perform home-based exercises three times a week. The primary outcome measure will be change from baseline to 12 weeks in the patient-reported outcome Disabilities of the Arm, Shoulder and Hand (DASH) questionnaire.

Discussion: Previous studies of exercise treatment for SIS have not differentiated between subgroups of SIS and have often had methodological flaws, making it difficult to specifically design target treatment for patients diagnosed with SIS. Therefore, it was considered important to focus on a subgroup such as tendinopathy, with a specific tailored intervention strategy based on evidence from other regions of the body, and to clearly describe the intervention in a methodologically strong study.
\end{abstract}

Trial registration: The trial was registered with Clinicaltrials.gov (NCT01984203) on 31 October 2013.

Keywords: Shoulder, Impingement, Tendinopathy, Exercise, Rotator cuff

\footnotetext{
* Correspondence: kingwersen@health.sdu.dk

${ }^{1}$ Institute of Sports Science and Clinical Biomechanics, University of Southern

Denmark, Campusvej 55, DK-5230 Odense, Denmark

2Physiotherapy Department, Hospital Lillebaelt, Vejle Hospital, Kabbeltoft 25,

DK-7100 Vejle, Denmark

Full list of author information is available at the end of the article
}

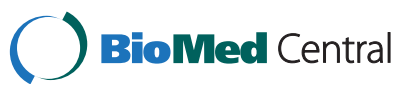

(c) 2015 Ingwersen et al.; licensee BioMed Central. This is an Open Access article distributed under the terms of the Creative Commons Attribution License (http://creativecommons.org/licenses/by/4.0), which permits unrestricted use, distribution, and reproduction in any medium, provided the original work is properly credited. The Creative Commons Public Domain Dedication waiver (http://creativecommons.org/publicdomain/zero/1.0/) applies to the data made available in this article, unless otherwise stated. 


\section{Background}

People with pain in the neck and shoulder region are often disabled to the point where they cannot live a normal life. The pain may also influence the person's work capacity and financial as well as social situation. Pain in the shoulder is the third most common musculoskeletal disorder, and the lifetime prevalence is estimated to be between seven and 10\% [1]. The prevalence for daily non-specific shoulder pain in a normal working population in an industrialised country is estimated to be $12 \%$ [2]. Furthermore, up to $23 \%$ of the workforce seeking care from the healthcare system with a shoulder problem have been sick-listed for more than one week [3].

Clinically, the most common shoulder-related cause of contact with the healthcare system is subacromial impingement syndrome (SIS), which accounts for 33\% of all shoulder-related healthcare contact [4,5]. SIS is generally considered to be a cluster of symptoms, rather than a single pathology. The Complaints of the Arm, Neck and/or Shoulder (CANS) model defines SIS as 'Disorders that include the rotator cuff syndrome, tendonitis of the $\mathrm{m}$. infraspinatus, $\mathrm{m}$. supraspinatus and $\mathrm{m}$. subscapularis, and bursitis in the shoulder area' [6]. The cause of SIS is considered to be multi-factorial and involves an altered muscle recruitment pattern in the rotator cuff and thoracoscapular muscles, potentially in combination with alterations to the anatomical structures in the subacromial space. These alterations may result in varying degrees of micro-traumas and degenerative pathophysiological changes in the rotator cuff muscle and surrounding tissue, which eventually can result in rotator cuff tendinopathy. Generally, the clinically relevant pathophysiological conditions associated with the diagnosis of SIS, such as rotator cuff tendinopathy, are often difficult to identify objectively [7,8], and consequently the diagnosis of rotator cuff tendinopathy is primarily based on a combination of the patient's history, clinical tests and ultrasonographic visualisation of changes in the rotator cuff tendons.

The Danish national clinical guidelines for the treatment of SIS recommend active rehabilitation strategies, and in cases where three to six months of exercise has been ineffective, arthroscopic subacromial decompression surgery can be considered [9]. These recommendations are, amongst others, based on the evidence that exercise has shown results comparable to arthroscopic subacromial decompression when evaluated on pain and patient-reported function [10-14]. However, the recommended guidelines rarely describe specific exercise programmes, nor are they sufficient in detail to be targeted at subgroups of SIS, such as rotator cuff tendinopathy. This means there is no consensus on specific rehabilitation exercise programmes for SIS patients with rotator cuff tendinopathy.
Among patients with Achilles or patella tendinopathy, studies of eccentric or progressive high-load exercises have shown promising effects on pain and function [15], and such exercises for chronic tendinopathy are often considered to be the first line of treatment [16-18]. Some of the mechanisms believed to be responsible for the positive outcomes of eccentric exercises are reduced structural abnormalities, such as hypoechoic areas, irregular structures, neovascularisation and tendon thickness $[19,20]$. The reasons for these changes could possibly be increased collagen type I synthesis [21]. Considering that collagen synthesis peaks at between 24 and 72 hours post-exercise [22], programmes prescribing daily training, such as those recommended for standard eccentric exercise programs, might not be optimal [23]. In support of this, very highload eccentric training with relatively few repetitions and low frequency (twice a week) has shown equally beneficial effects on patella tendinopathy $[24,25]$. Also, concentric heavy slow resistance exercises, without emphasis on the eccentric component, three times a week [25] has shown positive effects on patella tendinopathy. Furthermore, heavy slow resistance exercises seem to reduce abnormal fibrillar morphology among patients with patella tendinopathy [26].

However, rotator cuff tendinopathy is often treated as any other subgroup of SIS, with a combination of lowload exercises for internal and external rotation of the shoulder joint, with an attempt to target the rotator cuff muscles and optimise muscle coordination. Only a few studies have used progressive exercises or eccentric exercises in patients with SIS, and they have reported positive results on pain and function [27-32]. But all these studies have primarily targeted SIS and did not differentiate between the underlying pathologies. Inclusion of different subgroups of SIS in the same trial makes it difficult to customise an optimal exercise programme and, possibly, to detect a clinically relevant change between different exercise strategies [8].

Based on the evidence reported from studies on knee, Achilles and elbow tendons, applying eccentric or highload exercise programmes in patients with rotator cuff tendinopathy could be beneficial for patients' pain, function and quality of life [8], and for ultrasonographic visualised structural changes [33-35], when compared with traditional exercise strategies. But the rationale for using progressive high-load exercises is the diagnosis of tendinopathy and therefore, unlike existing studies, a study of eccentric or progressive high-load exercises should include only patients with tendinopathy. To our knowledge, no studies have investigated the effect on pain and function of progressive high-load exercises compared with traditional low-load exercises in the subgroup of SIS patients primarily showing signs of rotator cuff tendinopathy. A proportion of patients diagnosed 
with tendinopathy will also have a concomitant pathology in the surrounding tissue of the glenohumeral joint. Often corticosteroid injection into the subacromial space is used as an adjunct treatment for exercise interventions, if regarded relevant by the orthopaedic specialist. It is uncertain if this concomitant treatment has an additional effect on the exercise treatment.

The aim of the study is to determine in a randomised, double-blind controlled trial if a progressive high-load exercise programme for the rotator cuff muscles is superior to a traditional low-load exercise programme in patients with rotator cuff tendinopathy, based on the primary endpoint; change in score on the Disabilities of the Arm, Shoulder and Hand (DASH) questionnaire [36] after 12 weeks of exercise. The second aim is to evaluate the possible interaction of concomitant use of corticosteroids with the exercise intervention.

\section{Methods/Design}

\section{Study design}

This trial, called the Rotator Cuff Tendinopathy Exercise (RoCTEx) trial, is a multicentre (three sites), stratified (by corticosteroid injection (yes or no)), randomised, controlled, observer- and patient-blinded superiority trial, with a two-group parallel design, to be conducted in Denmark. The primary endpoint will be 12 weeks after baseline. Patients will be randomised to either progressive high-load exercises or low-load exercises (block randomisation with a 1:1 allocation). As illustrated in Figure 1, patients will also be assessed at one year from the baseline, referred to as the follow-up assessment.

\section{Settings and locations}

Patients will be recruited from the shoulder units at the outpatient clinics of orthopaedic departments at the Hospital Lillebaelt - Vejle Hospital, Aalborg University Hospital - Farsø Hospital and Odense University Hospital Svendborg Hospital.

\section{Participants}

The orthopaedic specialists will perform a standardized initial screening of all patients referred to the shoulder units. If patients fulfil the eligibility criteria they will be referred to the principal investigator, who will perform the final eligibility assessment and give detailed information about the study. Patients will be invited to participate if they meet the following inclusion criteria: between 18 and 65 years of age, have a current shoulder complaint lasting at least three months prior to the time of enrolment, pain is located in the proximal lateral aspect of the upper arm (C5 dermatome) that is aggravated by abduction, a positive 'Full Can Test' and/or Jobe's test and/or Resisted External Rotation Test, a positive Hawkins-Kennedy test and/or Neer's test, ultrasonographic verification of either tendon swelling, and/or presence of hypoechoic areas, calcification, fibrillar disruption and/or neovascularisation in the $\mathrm{m}$. supraspinatus.

Patients will be excluded if they fulfil any of the following exclusion criteria: Resting pain more than $40 \mathrm{~mm}$ on a visual analogue pain scale (range: 0 to 100), bilateral shoulder pain, less than 90 degrees of active elevation of the arm, a full thickness rupture in the rotator cuff tendon verified by ultrasonography, presence of calcification larger than $5 \mathrm{~mm}$ (vertical distance) verified by $\mathrm{x}$-ray, corticosteroid injection within the last six weeks, a radiologically verified fracture, glenohumeral osteoarthritis, prior surgery or dislocation of the affected shoulder, a clinically suspected labrum lesion, symptomatic arthritis in the acromioclavicular joint, a frozen shoulder or symptoms derived from the cervical spine, sensory or motor deficit in the neck or arm, suspected competing diagnoses (for example, rheumatoid arthritis, cancer, neurological disorders, fibromyalgia, psychiatric illness), pregnancy or inability to understand spoken and written Danish.

\section{Interventions}

Generally, both groups will receive the same exercises (although they differ on the amount of load), attention and basic information about ergonomic corrections related to the daily use of the upper extremity. The exercise program will consist of six exercises: two exercises for the scapula stabilising muscles (push-up plus exercise and low row exercise), two for the rotator cuff muscles (scaption and side-lying external rotation), and two stretching and mobility exercises (posterior capsule stretch and scapulae retraction). Patients will be instructed in performing home-based training three times per week, and will be seen for initial instruction in week one (60 minutes), and follow-up exercise sessions (30 minutes) in weeks two, three, four, six and nine. The general information about the project and exercise instructions will be given through a DVD to minimise the influence of the physiotherapist. On the first visit to the physiotherapist, the patients will receive detailed instructions on how to perform the exercise programme based on the instructions on the DVD. At the follow-up exercise sessions corrections will be made, and weights adjusted. The patients will be given a copy of the DVD and an exercise brochure to enable them to follow the instructions and exercises at home.

The difference between the intervention and control groups will be the external load targeting exercises for the rotator cuff. The intervention group will perform 12 weeks of progressive high-load exercises, gradually increasing the load from 15 repetitions maximum (RM) in week one to six RM in weeks nine to 12 . The intervention group will only be allowed to do isometric exercises if the pain exceeds $50 \mathrm{~mm}$ on the visual analogue scale. The control group will perform 12 weeks of low- 


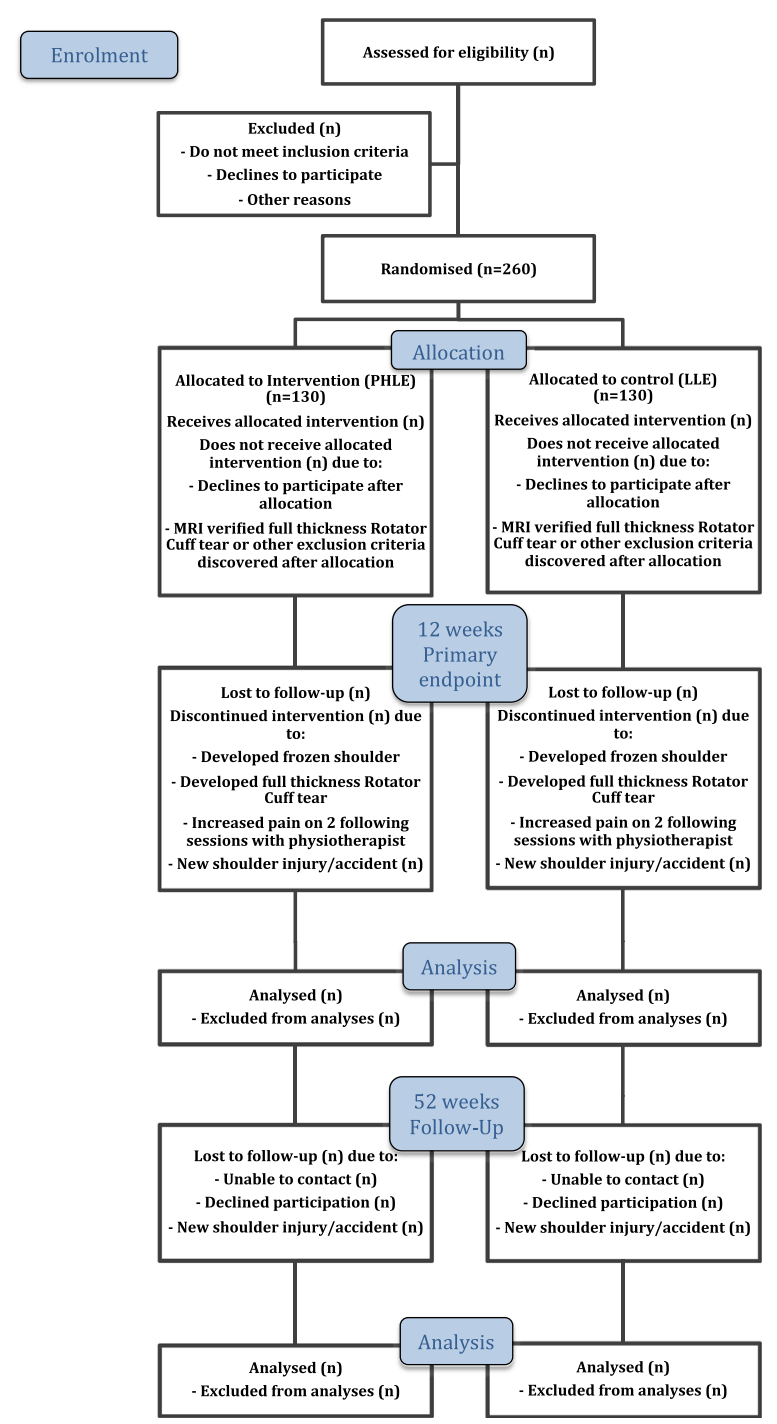

Figure 1 Expected flow of participants through the study. LLE: low-load exercises; PHLE: Progressive high-load exercises.

load exercises, performed with 20 RM from weeks one to 12 . For both groups, scapulae exercises will be performed with $20 \mathrm{RM}$, and stretching and mobility exercises will be performed five times, for 20 seconds each, in weeks one to 12 .

Concomitant use of corticosteroid injections will be based on the orthopaedic specialist's evaluation, in accordance with the department's standard procedures. Between 25 and $50 \%$ of patients are expected to receive injections. The corticosteroid injections will be given to patients at the shoulder unit of the orthopaedic departments, after baseline assessment but before randomisation. Randomisation will be performed in a stratified manner to ensure even distribution of patients receiving and not receiving corticosteroid injections in the two exercise groups.

\section{Outcome measures}

The primary outcome measure will be change from baseline to week 12 in the patient-reported outcome Disabilities of the Arm, Shoulder and Hand (DASH) questionnaire ( 0 to 100 score, $100=$ no problems) [36], and reported as the difference in mean change between the groups. The secondary endpoint assessment on the primary outcome will be performed 12 months after baseline (Figure 1).

The secondary outcome, measured at baseline and after 12 weeks, will include: the Shoulder injury and Osteoarthritis Outcome Score (SOOS; 0 to 100 score, $100=$ no problems) [37], the Hospital Anxiety and Depression Scale (HAD Scale; 0 to 21 score, $0=$ no problems) [38], EuroQol instrument (EQ-5D index; -0.59 to 1 score, $-0.59=$ lowest health-related quality of life), EuroQol Visual Analogue 
Scale (EQ-VAS; 0 to 100 score, $0=$ lowest health-related quality of life) [39], perceived pain at rest, general activity and maximum pain experienced during the previous 24 hours (visual analogue scale, 0 to 100 score, $0=$ no pain) [40], maximum isometric voluntary contraction (MVC) of external and/or internal rotation and scaption measured by dynamometers, active and passive range of movement in scaption ( 0 to 180 degrees), external ( 0 to 90 degrees) and internal rotation (0 to 90 degrees) measured by goniometer, clinical tests (Scapula Assistance Test [41], Scapula Retraction Test (positive or negative) [42] and ultrasonography measurements (aggregated sum of: supraspinatus tendon thickening (grade 0 to 3), fibrillar disruption (grade 0 to 3 ), calcification (grade 0 to 3 ) and neovascularisation (grade 0 to 4) [33]).

The following secondary outcomes will only be measured at the 12-month follow-up assessment: number of patients referred for, or completed, arthroscopic subacromial decompression; patients given subacromial corticosteroid injections after the intervention period; the number of visits to a general practitioner or secondary healthcare setting due to the shoulder problem (registration in the Danish Central Office of Patient Registration); and economic variables (for example, patient-reported number of sick days from work and sport attributed to the shoulder and time spent on rehabilitation in the trial period and post-trial, (patient-reported and registercontrolled). Table 1 presents all outcome measures and at which time point they will be collected.

Demographic data and other measurements will be recorded, covering socio-economic variables such as: employment, education, statement of income, history of injury (for example, accident, slow development and fluctuations), duration of current symptoms and physical demands in work and/or hobby activities (patient-reported).

The patients will be instructed to complete an exercise and pain diary. In the exercise diary, the patients will be asked to report the number of sets and repetitions per set for every training session, as well as the load during the exercise. Self-reported pain will be registered immediately before and 30 minutes after each training session. All pain measurements will be reported on the Numeric Pain Rating Scale. In addition to pain, patients will be asked to report use of analgesics (type and amount).

Good compliance will be defined by attendance at four out of the six visits to the physiotherapist, and an $80 \%$ complete exercise diary, where $100 \%$ will be defined by performance of 36 sessions of training (12 weeks at three training sessions per week).

\section{Data collection}

Three outcome assessors will perform all enrolment, baseline and follow-up assessments. Before starting the data collection, the assessors will train together with the primary investigator and decide on a consensus standard of interpreting all outcome variables.

\section{Sample size and power considerations}

Studies on patients with SIS have shown mean baseline values for DASH scores between 18 and 65, and standard deviations (SD) between 12 and 20 [30,31,43-47]. Often a $50 \%$ reduction in DASH score is seen after treatment $[30,31,44,46,47]$, and it is suggested that a $40 \%$ change corresponds to a substantial improvement for the patient [46]. Based on these data, we expect a mean baseline of 40 points on the DASH questionnaire with an SD of 17 . We expect a $50 \%$ change (change from 40 to $20 \mathrm{DASH}$ points) in the progressive high-load exercise group, and 25\% (change from 40 to $30 \mathrm{DASH}$ points) in the low-load exercise group (Figure 2A).

For a two-sample pooled t-test of a normal mean difference with a two-sided significance level of 0.05 $(P \leq 0.05)$, assuming a common SD of $17 \mathrm{DASH}$ points, a sample size of 100 patients per group has a power of 0.985 ( $>95 \%$ power for the primary outcome) to detect a between-group mean difference of 20 and 30 DASH points after 12 weeks in the progressive high-load exercise group and the low-load exercise group, respectively.

As the second objective is to explore the interaction between corticosteroid use (yes or no) and exercise (progressive high-load exercise or low-load exercise), it is hypothesised that, in the high-load group receiving corticosteroids at baseline, the DASH score at endpoint (12 weeks) will be more favourable than those without corticosteroids. With an SD of 17 DASH points, a sample size of 62 patients per stratum (having progressive high-load exercise) will be required to obtain a power of at least $0.9(90 \%)$ to detect a mean difference of $10 \mathrm{DASH}$ points between the two subgroups within the progressive high-load exercise group (Figure 2B). Consequently, it is decided to include 260 patients in the study, assuming a similar number of patients injected and not injected with corticosteroids $(1: 1 ; 2 \times 130$ patients randomly assigned to each exercise group (progressive high-load exercise versus lowload exercise)).

A final deadline for patient recruitment is set at Marts 2015, meaning that patient recruitment stops when the total number of 260 patients has been recruited, or the deadline of Marts 2015 is reached. In case the target number of 260 patients has not been met within the specified recruitment period, the power of the study will be less than expected. With an SD of 17 DASH points, a sample size of 46 patients per group will be required to obtain a power of at least $0.8(80 \%)$ to detect a mean difference of $10 \mathrm{DASH}$ points between the two groups. 
Table 1 Summary of measures to be collected

Socio-demographic measurements

$\begin{array}{ll}1,2,3 & \text { Age - year } \\ 1,2,3 & \text { Female sex - number (\%) } \\ 1,2,3 & \text { Duration of symptoms - year } \\ 1,2,3 & \text { Symptom history } \\ 1,2,3 & \text { - Accident or acute incidence } \\ 1,2,3 & \text { - Slow consistent development (overload) } \\ 1,2,3 & \text { - Fluctuating development } \\ 1,2,3 & \text { Compliance with exercise protocol } \\ 1,2,3 & \text { - Visits to the physiotherapy department }\end{array}$

$\begin{array}{llllll}\text { Yes } & \text { n/a } & \text { n/a } & n / a & n / a & n / a \\ \text { Yes } & n / a & n / a & n / a & n / a & n / a \\ \text { Yes } & n / a & n / a & n / a & n / a & n / a\end{array}$

Patient reported and physiological measurements

$\begin{array}{ll}1,2,3 & \text { Disabilities of the Arm, Shoulder and Hand (DASH) } \\ & \text { questionnaire (range: } 0 \text { to } 100)[36] \\ 1,2 & \text { Shoulder injury and Osteoarthritis Outcome Score (SOOS) [37] } \\ 1,2 & \text { Hospital Anxiety and Depression Scale (HAD Scale, } \\ & \text { range: } 0 \text { to } 21 \text { ) [38] } \\ 1,2 & \text { Visual analogue pain scale (VAS, range: } 0 \text { to 100) [40] } \\ & - \text { Rest } \\ & - \text { Maximum } \\ & - \text { Activity } \\ & \text { Isometric strength }\end{array}$

$\begin{array}{ll}1,3 & \text { - Scaption } \\ 1,3 & \text { - External rotation } \\ 1,3 & \text { - Internal rotation } \\ & \text { Degree of active movement }\end{array}$

$\begin{array}{llllll}\text { Yes } & \text { n/a } & \text { n/a } & \text { n/a } & \text { Yes } & \text { n/a } \\ \text { Yes } & \text { n/a } & \text { n/a } & \text { n/a } & \text { Yes } & \text { n/a } \\ \text { Yes } & \text { n/a } & \text { n/a } & \text { n/a } & \text { Yes } & \text { n/a }\end{array}$

1,3 - Scaption

1,3 - External rotation

1,3 - Internal rotation

Positive clinical test - number (\%)

$\begin{array}{llllll}\text { Yes } & \text { n/a } & \text { n/a } & \text { n/a } & \text { Yes } & \text { n/a } \\ \text { Yes } & n / a & n / a & n / a & \text { Yes } & \text { n/a } \\ \text { Yes } & \text { n/a } & n / a & n / a & \text { Yes } & n / a\end{array}$

$\begin{array}{llllll}\text { Yes } & \text { n/a } & \text { n/a } & \text { n/a } & \text { Yes } & \text { n/a } \\ \text { Yes } & \text { n/a } & \text { n/a } & \text { n/a } & \text { Yes } & \text { n/a } \\ \text { Yes } & \text { n/a } & \text { n/a } & \text { n/a } & \text { Yes } & \text { n/a }\end{array}$
$3 \quad$ - SAT [41]
$3 \quad-$ SRT [42]
Ultrasonographic measurement [33]
- Hypoechoic scale (range: 0 to 3)
$1 \quad$ - Neovascularity scale (range: 0 to 4 )
$\begin{array}{ll}1 & - \text { Tendon swelling }-\mathrm{mm} \\ 1 & \text { - Calcification (range: } 0 \text { to } 3 \text { ) }\end{array}$
$\begin{array}{ll}1 & - \text { Tendon swelling }-\mathrm{mm} \\ 1 & \text { - Calcification (range: } 0 \text { to } 3 \text { ) }\end{array}$

Yes

Yes

$\begin{array}{llllll}\text { Yes } & \text { n/a } & \text { n/a } & \text { n/a } & \text { n/a } & \text { n/a } \\ \text { Yes } & \text { n/a } & \text { n/a } & \text { n/a } & n / a & n / a \\ \text { Yes } & \text { n/a } & \text { n/a } & \text { n/a } & n / a & n / a\end{array}$

$n / a \quad n / a \quad n / a \quad n / a \quad$ Yes $n / a$

Socio-economic measurements

\section{Referred to}
1,2
- Operation - number (\%)
1,2
- Corticosteroid injection(s) - number

$\begin{array}{ll}n / a & n / a \\ n / a & n / a\end{array}$

$\begin{array}{ll}n / a & \text { Yes } \\ n / a & \text { Yes }\end{array}$

Yes

Yes 
Table 1 Summary of measures to be collected (Continued)

\begin{tabular}{|c|c|c|c|c|c|c|c|}
\hline & Shoulder related & & & & & & \\
\hline 2 & - Visits to general practitioner - number & $\mathrm{n} / \mathrm{a}$ & $\mathrm{n} / \mathrm{a}$ & $\mathrm{n} / \mathrm{a}$ & $\mathrm{n} / \mathrm{a}$ & Yes & Yes \\
\hline 2 & - Visits to secondary healthcare setting - number & n/a & $\mathrm{n} / \mathrm{a}$ & $\mathrm{n} / \mathrm{a}$ & $\mathrm{n} / \mathrm{a}$ & Yes & Yes \\
\hline 2 & - Sick days - number & $\mathrm{n} / \mathrm{a}$ & $\mathrm{n} / \mathrm{a}$ & $\mathrm{n} / \mathrm{a}$ & $\mathrm{n} / \mathrm{a}$ & Yes & Yes \\
\hline 2 & Time spent on shoulder-related rehabilitation - hours & $\mathrm{n} / \mathrm{a}$ & $\mathrm{n} / \mathrm{a}$ & $\mathrm{n} / \mathrm{a}$ & $\mathrm{n} / \mathrm{a}$ & Yes & Yes \\
\hline 2 & EuroQol-5D (EQ-5D, range: 0 to 100) [39] & Yes & $\mathrm{n} / \mathrm{a}$ & $\mathrm{n} / \mathrm{a}$ & $\mathrm{n} / \mathrm{a}$ & Yes & Yes \\
\hline
\end{tabular}

n/a: not assessed; SAT: Scapulae Assistance Test; SRT: Scapulae Retraction Test.

Article 1: Progressive high-load exercise compared with general low-load exercise in patients with rotator cuff tendinopathy: a randomised trial.

Article 2: The effect of 12 weeks of progressive high-load exercise in patients with rotator cuff tendinopathy on patient-reported and economic outcomes -

12 months follow-up.

Article 3: The prognostic evaluation of clinical tests in patients with rotator cuff tendinopathy.

\section{Randomisation and allocation concealment}

Patients will be randomly assigned to either of the two exercise groups with a 1:1 allocation as per a computergenerated randomisation schedule, stratified by administration of concomitant corticosteroid injection using permuted blocks of random sizes (two to six). The primary investigator, assessors and administrator of the randomisation procedure will not know block sizes in order to ensure allocation concealment. In practice, after recruitment and baseline measurements, the secretary at the physiotherapy department will administer the allocation procedure by taking a sequentially numbered, opaque sealed envelope from one of two ring binders, determined by the potential referral to corticosteroids (yes or no) by the orthopaedic specialist.

\section{Blinding}

Outcome assessors will perform both baseline and followup assessments, and will be kept blinded from treatment allocation. At the follow-up assessments, patients will be strongly encouraged not to disclose the components of their exercise programme, in order to keep the outcome assessor blinded. Patients will be blinded regarding exercise allocation by informing them that the trial involves testing two exercise programmes consisting of the same exercises, and that they will be performed in two different ways, but not defining the specific difference between these, or which is hypothesised to be more effective.

\section{Statistical analysis plan}

The primary efficacy analysis performed is assessment of the between-group difference in change in the DASH score after 12 weeks in the intent-to-treat (ITT) population (all randomised patients independent of compliance and withdrawals). In the case of missing data due to dropouts, a non-responder imputation will be applied; a baseline observation carried forward (BOCF) technique will be used for patients who do not complete the study (as will be illustrated in a corresponding trial flow diagram).

For the primary analyses at week 12, we will use analysis of covariance (ANCOVA) to compare the progressive high-load exercise group with the low-load exercise group for mean changes from baseline in the DASH score, as well as the secondary continuous outcomes. The primary model includes the change from baseline as the dependent variable, with treatment group (progressive high-load exercise or low-load exercise), corticosteroid status (yes or no), and the centre site (one, two or three) as main effects, with the baseline score as an additional covariate. The secondary analysis for the primary outcome will add the interaction term for group $\times$ corticosteroid in the primary model.

To analyse the longitudinal element of time effects of DASH in the randomised trial (repeated measures design three, six, nine, and 12 weeks), a linear approach will be used, fitted in SAS software (version 9.3 Service Pack 4; SAS Institute Inc., Cary, North Carolina, United States) using the procedure 'PROC MIXED' based on restricted maximum likelihood (REML) estimates of the parameters. The variable 'patient' will be applied as a random effects factor. Assessment of the treatment and time effects is of exploratory interest for the primary outcome in testing for a possible interaction, and both treatment and time will be used as systematic factors, using the baseline value as covariate to reduce random variation and increase power.

For all of the above analyses, results will be expressed as the difference between the group means and 95\% confidence intervals with associated $P$ values, based on the mixed linear model. All data analyses will be carried out according to the pre-established analysis plan, and will be performed applying SAS software. All descriptive statistics and tests will be reported in accordance with the recommendations of the Enhancing the QUAlity and Transparency Of health Research (EQUATOR) network [48] and the Consolidating Standards of Reporting Trials (CONSORT) statement [49]. In order to evaluate the empirical distributions of the continuous outcomes, visual inspection (of the studentised residuals from the statistical model) will be used to suggest whether the assumption of normality and variance homogeneity is reasonable. 

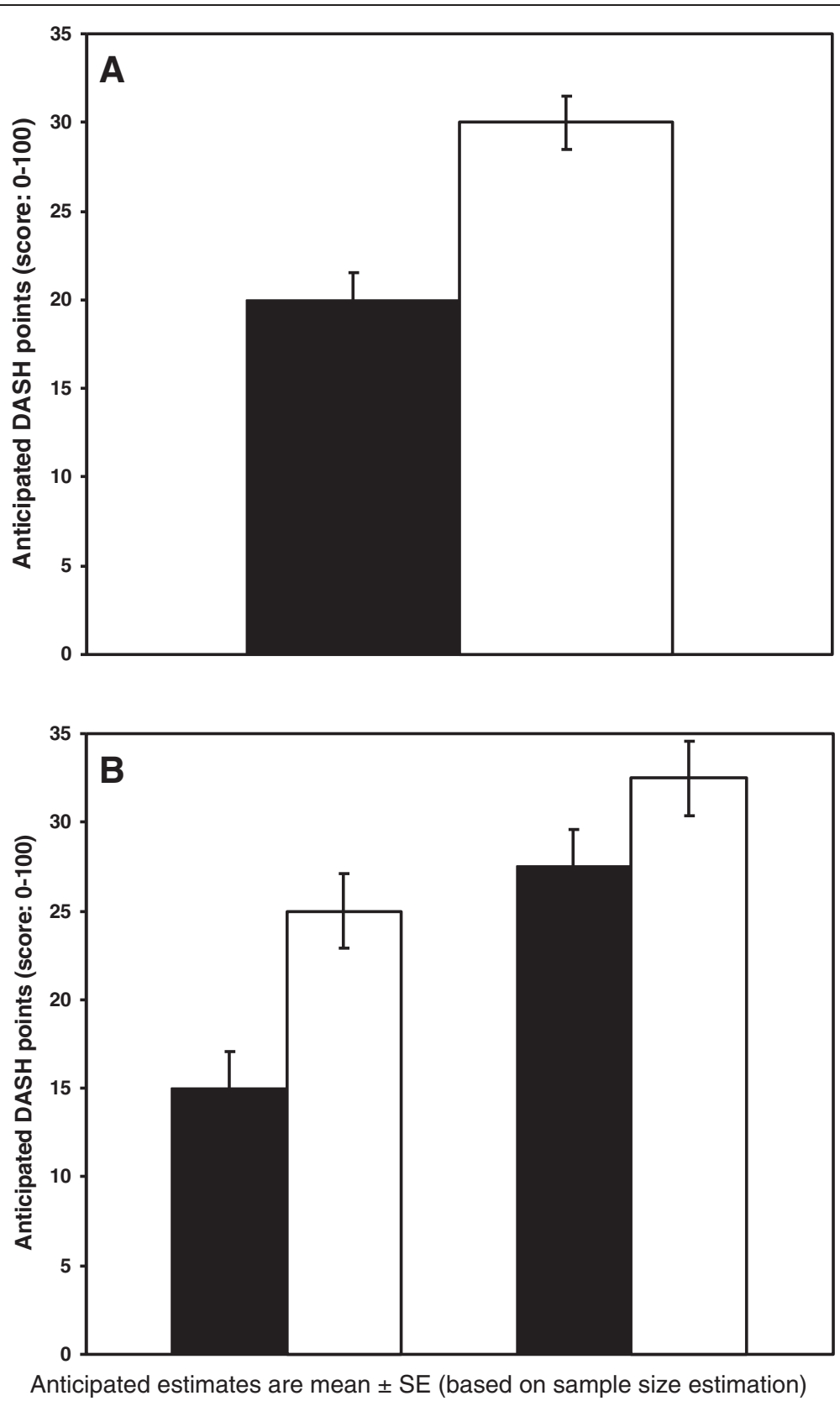

Figure 2 Anticipated outcome (primary and secondary objectives). A: (Primary objective) Illustration of expected DASH score at 12 weeks. Black = intervention group, White = control group. B: (Secondary objective) Illustration of expected DASH score at 12 weeks stratified for concomitant corticosteroid injection. Columns I+ II= intervention group, Columns III + IV = control group, Black= concomitant corticosteroid injection, White = no concomitant corticosteroid injection. DASH, Disabilities of the Arm, Shoulder and Hand Questionnaire.

Statistical analysis of the economic variables will be presented as descriptive statistics, and a cost-utility analysis of the economic burden of each specific exercise programme in relation to the EQ-5D will be performed, expressed in quality-adjusted life years (QALYs) gained.

\section{Interim analysis and early stopping rules}

An early stopping rule will be applied when complete rotator cuff ruptures, verified by ultrasonography, occur due to the performance of the exercise programme. If six or more cases of complete rupture occur in the progressive high-load exercise group, and the rate of rupture is $50 \%$ more compared with the low-load exercise group, the trial will be stopped.

\section{Data monitoring}

Since adverse events are expected to be minimal and the intervention is not considered a high-risk intervention, 
no data monitoring committee will be established. The physiotherapists at each centre will be asked to report adverse events to the primary investigator, who will report these to the ethics committee and monitor if the number of adverse events has reached the threshold for the early stopping rule.

\section{Ethics}

All patients will participate on a voluntary basis and will be informed that they can withdraw at any time without this influencing their subsequent treatment. Informed consent will be obtained from all patients. The Regional Scientific Ethics Committee of Southern Denmark approved the trial on the 27 June 2013 (project ID: S-20130071). The trial was registered with the Danish Data Protection Agency and approved on the 30 May 2013 (registration number: 2008-58-0035). The trial is registered at Clinicaltrials.gov (identifier: NCT01984203). The Danish Act concerning Processing of Personal Data (DAPPD) will be followed. The trial will follow the principles of the Declaration of Helsinki [50].

\section{Discussion}

The effect of exercise on the rehabilitation of SIS has been well studied, but the latest systematic reviews focusing on active exercise rehabilitation of SIS consistently report that most studies are of low to moderate quality [8,51-57]. This is primarily due to small sample sizes, inadequate blinding of patients and/or investigators and incomplete intervention descriptions, making it difficult to translate the results into clinical practice. Furthermore, previous studies show a wide range in clinical outcome (from some effect to almost no effect), which is probably the result of mixed populations within the SIS 'umbrella of pathologies', and variation in the interventions used [8]. However, collectively there is evidence that active exercise is effective in the rehabilitation of SIS. When evaluating new treatment approaches that already have some evidence of efficacy, it is considered unethical to compare treatment with a placebo or similar. New approaches should at least always be tested against current best practice.

The RoCTEx trial will add new knowledge to the field as described in the following points. Firstly, the focus on patients with rotator cuff tendinopathy will result in a more homogeneous group, giving the possibility to transfer exercise principles (such as progressive highload exercise) to the shoulder that previously have been shown to have positive results in other body regions. The specific exercises for this trial have been selected on the basis of evidence from Electromyography (EMG) studies, resulting in targeting primarily the $\mathrm{m}$. supraspinatus, which is most often considered the site of tendinopathy. In addition, the trial's exercises will be focusing on stabilising muscles for the scapulae, thereby providing the foundation for optimal functioning of the glenohumeral joint [58-61]. Secondly, efforts will be made to keep both primary assessors and patients blinded to the intervention group, which has been one of the most frequent criticisms of earlier studies. Thirdly, we will include an active rehabilitation strategy for our control group in order to specify if the effect of the intervention is due specifically to the high-load exercise, or merely to the effect of improved muscle activation of the shoulder muscles. Fourthly, allowing for a subgroup of the included patients having a corticosteroid injection if deemed necessary by the orthopaedic specialist, and subsequently stratifying for this effect, increases the external validity of the results.

Since no gold standard exists for diagnosing rotator cuff tendinopathy, the current inclusion criteria may be questioned. The inherent diagnostic difficulties could result in the inclusion of patients not having rotator cuff tendinopathy, thereby increasing participant variability and thereby make it more difficult to reach a significant result. However, our sample size is calculated on the basis of studies with the same variation in populations, thereby presumably taking this variation into account. Furthermore, ultrasound has shown moderate sensitivity and excellent specificity in diagnosing rotator cuff tendinopathy. The excellent specificity of 0.88 suggests an acceptable probability of not including patients who do not have rotator cuff tendinopathy [62].

The multifactorial pathophysiology of SIS (rotator cuff pathology and degeneration based on decreased vascularity, scapular dyskinesia, instability of the glenohumeral joint or glenohumeral internal rotation deficit) may result in patients not responding positively to a single modality exercise programme. However, as we focus our inclusion on those patients who have signs of rotator cuff tendinopathy, and only distinguish the intervention and control groups by the external load applied, we expect that this will not excessively dilute the results. Furthermore, the trial's exercise programme will consider the multifactorial origin by including exercises focused on the overall strength, stability and mobility of the scapula-humeral complex.

The progressive high-load exercise programme is optimised in several ways that distinguish it from traditional eccentric exercise programmes. The concentric heavy slow resistance exercises are favourable because of their similarity to daily activities, thereby presumably increasing the likelihood of compliance. Furthermore, eccentric exercises for the rotator cuff muscles may induce a more unsuitable initial position, reducing the subacromial space, with an anticipated increased risk of subacromial impingement. Alternatively, using exercises starting with a concentric phase, and allowing the 
patients only to do isometric exercises when restricted by pain, will potentially minimise the risk of irritating the subacromial structures.

The objective of the RoCTEx trial is to show whether there is a significant advantage in progressive high-load exercise compared with the more traditional low-load exercise. If this is the case, the description and availability of a standardised progressive high-load exercise programme will help practitioners to treat patients diagnosed with rotator cuff tendinopathy. If no difference occurs between the two groups, practitioners will have the possibility of allowing their patients to choose the exercise modality that best optimises their compliance.

\section{Trial status}

At the time of submission of this study protocol, the trial is ongoing and still recruiting patients. Recruitment began November 2013, and is expected to end Marts 2015.

\section{Abbreviations \\ ANCOVA: ANalysis of COVAriance; BOCF: Baseline Observation Carried Forward; CANS: Complaints of the Arm, Neck and/or Shoulder; CONSORT: CONsolidating Standards Of Reporting Trials; DAPPD: Danish Act concerning Processing of Personal Data; DASH: Disabilities of the Arm, Shoulder and Hand questionnaire; EMG: ElectroMyoGraphy; EQUATOR: Enhancing the QUAlity and Transparency Of health Research HAD: Hospital Anxiety and Depression Scale; ITT: Intention-To-Treat; LLE: Low-Load Exercises; MVC: Maximum isometric Voluntary Contraction; PHLE: Progressive High-Load Exercises; QUALY: QUality-Adjusted Life Years; RM: Repetitions Maximum; RC: Rotator Cuff; RoCTEx: Rotator Cuff Tendinopathy Exercise; SAT: Scapulae Assistance Test; SD: Standard Deviations; SIS: Subacromial Impingement Syndrome; SRT: Scapulae Retraction Test; SOOS: Shoulder injury and Osteoarthritis Outcome Score; VAS: Visual Analogue Scale.}

\section{Competing interests}

The authors declare that they have no competing interests.

\section{Authors' contributions}

$\mathrm{KGI}$ will be the primary assessor in the trial. He initiated the project in collaboration with BJK and KS, and will lead the coordination of the trial. KGI, $\mathrm{BJK}, \mathrm{KS}$ and RC assisted in trial design and wrote this manuscript. RC designed the statistical analysis plan and provided the randomisation schedule. KGI, LS, SLJ, SR and HJ planned the coordination at the hospitals. All authors were involved in drafting the manuscript and critically revising it for important intellectual content and, finally, read and approved the final manuscript.

\section{Acknowledgements}

We thank all participating orthopaedic specialists and physiotherapists performing the initial screening of patients and instructing patients in the exercises.

The participating departments at Hospital Lillebaelt - Vejle Hospital, Aalborg University Hospital - Farsø Hospital and Odense University Hospital - Svendborg Hospital will contribute personnel. Region of Southern Denmark's Research fund, The Danish Rheumatism Association and the Ryholts Foundation funds the trial. Dr Christensen is employed in the Musculoskeletal Statistics Unit at The Parker Institute, which is supported by grants from the Oak Foundation. We thank them for their support.

\section{Author details}

${ }^{1}$ Institute of Sports Science and Clinical Biomechanics, University of Southern Denmark, Campusvej 55, DK-5230 Odense, Denmark. ²Physiotherapy Department, Hospital Lillebaelt, Vejle Hospital, Kabbeltoft 25, DK-7100 Vejle, Denmark. ${ }^{3}$ Department of Rheumatology, Musculoskeletal Statistics Unit, The Parker Institute, Copenhagen University Hospital, Bispebjerg and
Frederiksberg, Nordre Fasanvej 57, road 8, entrance 19, DK-2000 Frederiksberg, Denmark. ${ }^{4}$ Orthopaedic Department, Shoulder Unit, Hospital Lillebaelt, Vejle Hospital, Kabbeltoft 25, DK-7100 Vejle, Denmark. ${ }^{5}$ Orthopaedic Department, Shoulder Unit, Odense University Hospital, Svendborg Hospital, Valdemarsgade 53, DK-5700 Svendborg, Denmark. ${ }^{6}$ Department of Orthopaedic Surgery, Shoulder Unit, Aalborg University Hospital, Fars $\varnothing$ Hospital, Højgårdsvej 11, DK-9640 Farsø, Denmark. ${ }^{7}$ Orthopaedic Surgery Research Unit, Aalborg University Hospital, Sdr. Skovvej 15, DK-9000 Aalborg, Denmark. ${ }^{8}$ Department of Clinical Medicine, Aalborg University, Sdr. Skovvej 15, DK-9000 Aalborg, Denmark. ${ }^{9}$ Department of Health Sciences, Institute of Occupational Therapy, Physiotherapy and Radiography, Bergen University

College, Inndalsveien 28, NO-5063 Bergen, Norway.

Received: 29 September 2014 Accepted: 23 December 2014

Published online: 27 January 2015

\section{References}

1. Luime JJ, Koes BW, Hendriksen IJ, Burdorf A, Verhagen AP, Miedema HS, et al. Prevalence and incidence of shoulder pain in the general population: a systematic review. Scand J Rheumatol. 2004;33:73-81.

2. Miranda H, Viikari-Juntura E, Heistaro S, Heliovaara M, Riihimaki H. A population study on differences in the determinants of a specific shoulder disorder versus nonspecific shoulder pain without clinical findings. Am J Epidemiol. 2005:161:847-55

3. Huisstede BM, Wijnhoven HA, Bierma-Zeinstra SM, Koes BW, Verhaar JA, Picavet S. Prevalence and characteristics of complaints of the arm, neck, and/or shoulder (CANS) in the open population. Clin J Pain. 2008;24:253-9.

4. Feleus A, Bierma-Zeinstra SM, Miedema HS, Verhaar JA, Koes BW. Management in non-traumatic arm, neck and shoulder complaints: differences between diagnostic groups. Eur Spine J. 2008;17:1218-29.

5. van der Windt DA, Koes BW, de Jong BA, Bouter LM. Shoulder disorders in general practice: incidence, patient characteristics, and management. Ann Rheum Dis. 1995;54:959-64.

6. Huisstede BM, Miedema HS, Verhagen AP, Koes BW, Verhaar JA. Multidisciplinary consensus on the terminology and classification of complaints of the arm, neck and/or shoulder. Occup Environ Med. 2007;64:313-9.

7. Lewis JS. Rotator cuff tendinopathy/subacromial impingement syndrome: is it time for a new method of assessment? Br J Sports Med. 2009;43:259-64.

8. Littlewood C, Ashton J, Chance-Larsen K, May S, Sturrock B. Exercise for rotator cuff tendinopathy: a systematic review. Physiotherapy. 2012;98:101-9.

9. Authority DHaM. National clinical guidelines for diagnostic and treatment of patients with selected shoulder impairments. In: National clinical guidelines - publications. 2013. http://sundhedsstyrelsen.dk/publ/Publ2013/09sep/ NKRUdvalgteSkulderlidelser.pdf. Accessed 14 Jan 2015. Danish.

10. Brox Jl, Gjengedal E, Uppheim G, Bohmer AS, Brevik Jl, Ljunggren AE, et al. Arthroscopic surgery versus supervised exercises in patients with rotator cuff disease (stage II impingement syndrome): a prospective, randomized, controlled study in 125 patients with a 2 1/2-year follow-up. J Shoulder Elbow Surg. 1999;8:102-11.

11. Brox Jl, Staff PH, Ljunggren AE, Brevik Jl. Arthroscopic surgery compared with supervised exercises in patients with rotator cuff disease (stage II impingement syndrome). BMJ. 1993;307:899-903.

12. Haahr JP, Andersen JH. Exercises may be as efficient as subacromial decompression in patients with subacromial stage II impingement: 4-8-years' follow-up in a prospective, randomized study. Scand J Rheumatol. 2006;35:224-8

13. Haahr JP, Ostergaard S, Dalsgaard J, Norup K, Frost P, Lausen S, et al. Exercises versus arthroscopic decompression in patients with subacromial impingement: a randomised, controlled study in 90 cases with a one year follow up. Ann Rheum Dis. 2005;64:760-4.

14. Ketola S, Lehtinen J, Arnala I, Nissinen M, Westenius H, Sintonen $\mathrm{H}$, et al. Does arthroscopic acromioplasty provide any additional value in the treatment of shoulder impingement syndrome?: a two-year randomised controlled trial. J Bone Joint Surg Br. 2009;91:1326-34.

15. Malliaras $\mathrm{P}$, Barton CJ, Reeves ND, Langberg $\mathrm{H}$. Achilles and patellar tendinopathy loading programmes: a systematic review comparing clinical outcomes and identifying potential mechanisms for effectiveness. Sports Med. 2013;43:267-86.

16. Andres BM, Murrell GA. Treatment of tendinopathy: what works, what does not, and what is on the horizon. Clin Orthop Relat Res. 2008:466:1539-54. 
17. Cook JL, Purdam CR. Is tendon pathology a continuum? A pathology model to explain the clinical presentation of load-induced tendinopathy. Br J Sports Med. 2009;43:409-16.

18. Kingma JJ, de Knikker R, Wittink HM, Takken T. Eccentric overload training in patients with chronic Achilles tendinopathy: a systematic review. Br J Sports Med. 2007:41:e3.

19. Ohberg $L$, Alfredson $H$. Effects on neovascularisation behind the good results with eccentric training in chronic mid-portion Achilles tendinosis? Knee Surg Sports Traumatol Arthrosc. 2004;12:465-70.

20. Ohberg L, Lorentzon $\mathrm{R}$, Alfredson $\mathrm{H}$. Eccentric training in patients with chronic Achilles tendinosis: normalised tendon structure and decreased thickness at follow up. Br J Sports Med. 2004;38:8-11. discussion 11.

21. Langberg H, Ellingsgaard H, Madsen T, Jansson J, Magnusson SP, Aagaard P, et al. Eccentric rehabilitation exercise increases peritendinous type I collagen synthesis in humans with Achilles tendinosis. Scand J Med Sci Sports. 2007;17:61-6.

22. Miller BF, Olesen JL, Hansen M, Dossing S, Crameri RM, Welling RJ, et al. Coordinated collagen and muscle protein synthesis in human patella tendon and quadriceps muscle after exercise. J Physiol. 2005;567:1021-33.

23. Langberg $\mathrm{H}$, Kongsgaard $\mathrm{M}$. Eccentric training in tendinopathy-more questions than answers. Scand J Med Sci Sports. 2008;18:541-2.

24. Frohm A, Saartok T, Halvorsen K, Renstrom P. Eccentric treatment for patellar tendinopathy: a prospective randomised short-term pilot study of two rehabilitation protocols. Br J Sports Med. 2007;41:e7.

25. Kongsgaard M, Kovanen $V$, Aagaard $P$, Doessing $S$, Hansen $P$, Laursen AH, et al. Corticosteroid injections, eccentric decline squat training and heavy slow resistance training in patellar tendinopathy. Scand J Med Sci Sports. 2009;19:790-802.

26. Kongsgaard M, Qvortrup K, Larsen J, Aagaard P, Doessing S, Hansen P, et al. Fibril morphology and tendon mechanical properties in patellar tendinopathy: effects of heavy slow resistance training. Am J Sports Med. 2010;38:749-56.

27. Maenhout AG, Mahieu NN, De Muynck M, De Wilde LF, Cools AM. Does adding heavy load eccentric training to rehabilitation of patients with unilateral subacromial impingement result in better outcome? A randomized clinical trial. Knee Surg Sports Traumatol Arthrosc. 2013;21:1158-67.

28. Lombardi Jr I, Magri AG, Fleury AM, Da Silva AC, Natour J. Progressive resistance training in patients with shoulder impingement syndrome: a randomized controlled trial. Arthritis Rheum. 2008:59:615-22.

29. Jonsson P, Wahlstrom P, Ohberg L, Alfredson H. Eccentric training in chronic painful impingement syndrome of the shoulder: results of a pilot study. Knee Surg Sports Traumatol Arthrosc. 2006;14:76-81.

30. Holmgren T, Bjornsson Hallgren H, Oberg B, Adolfsson L, Johansson K. Effect of specific exercise strategy on need for surgery in patients with subacromial impingement syndrome: randomised controlled study. BMJ. 2012;344:e787.

31. Camargo PR, Avila MA, Alburquerque-Sendin F, Asso NA, Hashimoto LH, Salvini TF. Eccentric training for shoulder abductors improves pain, function and isokinetic performance in subjects with shoulder impingement syndrome: a case series. Rev Bras Fisioter. 2012;16:74-83.

32. Bernhardsson S, Klintberg $I H$, Wendt GK. Evaluation of an exercise concept focusing on eccentric strength training of the rotator cuff for patients with subacromial impingement syndrome. Clin Rehabil. 2011;25:69-78.

33. Poltawski L, Ali S, Jayaram V, Watson T. Reliability of sonographic assessment of tendinopathy in tennis elbow. Skeletal Radiol. 2012:41:83-9.

34. Kim SY, Ko JB, Farthing JP, Butcher SJ: Investigation of supraspinatus muscle architecture following concentric and eccentric training. J Sci Med Sport. 2014. doi: 10.1016/j.jsams.2014.05.007.

35. Kim S, Bleakney R, Boynton E, Ravichandiran K, Rindlisbacher T, McKee N, et al. Investigation of the static and dynamic musculotendinous architecture of supraspinatus. Clin Anat. 2010:23:48-55.

36. Hudak PL, Amadio PC, Bombardier C. Development of an upper extremity outcome measure: the DASH (disabilities of the arm, shoulder and hand) [corrected]. The Upper Extremity Collaborative Group (UECG). Am J Ind Med. 1996;29:602-8.

37. Ingwersen KG BJ-K. Shoulder Injury and Osteoarthritis Outcome Score (SOOS) - Reliability and Validity of a New Patient Reported Outcome Measure. In: Sports Medicine Congress; 2012 Feb 2-4; Kolding, Denmark. Kolding, Denmark: Dansk Idrætsmedicinsk Selskab; 2012.

38. Zigmond AS, Snaith RP. The hospital anxiety and depression scale. Acta Psychiatr Scand. 1983;67:361-70.

39. Rabin R, de Charro F. EQ-5D: a measure of health status from the EuroQol Group. Ann Med. 2001;33:337-43.

40. Carlsson AM. Assessment of chronic pain. I. Aspects of the reliability and validity of the visual analogue scale. Pain. 1983;16:87-101.
41. Kibler WB. The role of the scapula in athletic shoulder function. Am J Sports Med. 1998;26:325-37.

42. Kibler WB, McMullen J. Scapular dyskinesis and its relation to shoulder pain. J Am Acad Orthop Surg. 2003;11:142-51.

43. de Witte PB, Henseler JF, Nagels J, Vliet Vlieland TP, Nelissen RG. The Western Ontario rotator cuff index in rotator cuff disease patients: a comprehensive reliability and responsiveness validation study. Am J Sports Med. 2012;40:1611-9.

44. Karthikeyan S, Kwong HT, Upadhyay PK, Parsons N, Drew SJ, Griffin D. A double-blind randomised controlled study comparing subacromial injection of tenoxicam or methylprednisolone in patients with subacromial impingement. J Bone Joint Surg Br. 2010;92:77-82.

45. Lee JH, Lee SH, Song SH. Clinical effectiveness of botulinum toxin type B in the treatment of subacromial bursitis or shoulder impingement syndrome. Clin J Pain. 2011;27:523-8.

46. Michener LA, Snyder Valier AR, McClure PW. Defining substantial clinical benefit for patient-rated outcome tools for shoulder impingement syndrome. Arch Phys Med Rehabil. 2013;94:725-30.

47. Tate AR, McClure PW, Young IA, Salvatori R, Michener LA. Comprehensive impairment-based exercise and manual therapy intervention for patients with subacromial impingement syndrome: a case series. J Orthop Sports Phys Ther 2010:40:474-93.

48. Christensen R, Bliddal H, Henriksen M. Enhancing the reporting and transparency of rheumatology research: a guide to reporting guidelines. Arthritis Res Ther. 2013;15:109.

49. Moher D, Hopewell S, Schulz KF, Montori V, Gotzsche PC, Devereaux PJ, et al. CONSORT 2010 explanation and elaboration: updated guidelines for reporting parallel group randomised trials. J Clin Epidemiol. 2010;63:e1-37.

50. Vollmann J, Winau R. Informed consent in human experimentation before the Nuremberg code. BMJ. 1996;313:1445-9.

51. Faber E, Kuiper Jl, Burdorf A, Miedema HS, Verhaar JA. Treatment of impingement syndrome: a systematic review of the effects on functional limitations and return to work. J Occup Rehabil. 2006;16:7-25.

52. Kelly SM, Wrightson PA, Meads CA. Clinical outcomes of exercise in the management of subacromial impingement syndrome: a systematic review. Clin Rehabil. 2010;24:99-109.

53. Kromer TO, Tautenhahn UG, de Bie RA, Staal JB, Bastiaenen $\mathrm{CH}$. Effects of physiotherapy in patients with shoulder impingement syndrome: a systematic review of the literature. J Rehabil Med. 2009;41:870-80.

54. Kuhn JE. Exercise in the treatment of rotator cuff impingement: a systematic review and a synthesized evidence-based rehabilitation protocol. J Shoulder Elbow Surg. 2009;18:138-60.

55. Michener LA, Walsworth MK, Burnet EN. Effectiveness of rehabilitation for patients with subacromial impingement syndrome: a systematic review. J Hand Ther. 2004;17:152-64.

56. Papadonikolakis A, McKenna M, Warme W, Martin BI, Matsen 3rd FA. Published evidence relevant to the diagnosis of impingement syndrome of the shoulder. J Bone Joint Surg Am. 2011;93:1827-32.

57. von der Heyde RL. Occupational therapy interventions for shoulder conditions: a systematic review. Am J Occup Ther. 2011;65:16-23.

58. Andersen $\mathrm{CH}$, Zebis MK, Saervoll C, Sundstrup E, Jakobsen MD, Sjogaard G, et al. Scapular muscle activity from selected strengthening exercises performed at low and high intensities. J Strength Cond Res. 2012;26:2408-16.

59. Cools AM, Dewitte V, Lanszweert F, Notebaert D, Roets A, Soetens $B$, et al. Rehabilitation of scapular muscle balance: which exercises to prescribe? Am J Sports Med. 2007;35:1744-51.

60. Escamilla RF, Yamashiro K, Paulos L, Andrews JR. Shoulder muscle activity and function in common shoulder rehabilitation exercises. Sports Med. 2009:39:663-85.

61. Kibler WB, Sciascia AD, Uhl TL, Tambay N, Cunningham T. Electromyographic analysis of specific exercises for scapular control in early phases of shoulder rehabilitation. Am J Sports Med. 2008;36:1789-98.

62. Martin-Hervas C, Romero J, Navas-Acien A, Reboiras JJ, Munuera L. Ultrasonographic and magnetic resonance images of rotator cuff lesions compared with arthroscopy or open surgery findings. J Shoulder Elbow Surg. 2001;10:410-5. 